\title{
A Preliminary Trial of the Introduction of Computerized Decision Support to Assist Resuscitation of the Severely Injured in a Level 1 Trauma Centre in India
}

\author{
Mark C. Fitzgerald ${ }^{1} \cdot$ Amit Gupta $^{2} \cdot$ Sanjeev Kumar Bhoi ${ }^{2} \cdot$ Yesul Kim $^{1}$ (D) $\cdot$ Ankita Sharma $^{2} \cdot$ Ashish Jhakal $^{2}$. \\ Joseph Mathew ${ }^{1} \cdot$ Mahesh Chandra Misra ${ }^{2}$
}

Received: 21 September 2020 / Accepted: 7 January 2021 / Published online: 24 February 2021

(C) The Author(s) 2021

\begin{abstract}
Injury from motor vehicle accidents remains a leading cause of death in India with increasing number of fatalities. Timely delivery of lifesaving interventions is critical for survival and in restoring physical functioning. As a part of the Australia India Trauma Systems Collaboration, the Trauma Reception and Resuscitation (TRR@)-computerized decision support system was implemented in a Level 1 Trauma Centre in India in order to determine whether this system would reduce the time in performing lifesaving interventions and improve vital sign data capture and documentation. This prospective cohort study at the Jai Prakash Narayan Apex Center, All India Institute of Medical Science, New Delhi, recruited a total of 106 participants into two groups: TRR@ (76) and controls (30). During the first $30 \mathrm{~min}$ of resuscitation, the TRR@ group recorded greater sets of vital signs in compared to the controls for medical records. More importantly, the real-time documentation of the vital signs for the TRR@ group ensured accuracy for medical records. For lifesaving interventions, oxygen was administered in the TRR@ group only if $\mathrm{SpO}_{2}<93 \%$, whereas oxygen was administered as standard of care in the controls. There was no statistical difference in the mean times to endotracheal intubation, intercostal catheter insertion or performance of emergency chest $\mathrm{x}$-ray between the control and TRR@ groups. Importantly however, these 3 comparable interventions were performed consistently within a smaller timeframe for patients receiving care with TRR@ decision prompts. There was a greater variability in the time taken to perform lifesaving interventions in the control group in comparison to the clinicians assisted with computerized decision prompts. This preliminary study was not powered to measure difference in mortality and patient recruitment was limited to $8 \mathrm{am}-5 \mathrm{pm}$ when trained staff could attend to operating the TRR@ system.
\end{abstract}

Keywords Decision support $\cdot$ Resuscitation $\cdot$ Trauma $\cdot$ Lifesaving interventions $\cdot$ Vital sign documentation

\section{Introduction}

Over 5 million people worldwide die each year following injury [1]. More than one death in every ten occurs in India, where tragedy on the roads, and in workplaces, is increasing. Many survivors are disabled by their injuries, and more productive years of life are lost following injury than any other cause.

Yesul Kim

yen.kim@alfred.org.au

1 National Trauma Research Institute, 89 Commercial Road, Melbourne, VIC 3004, Australia

2 Jai Prakash Narayan Apex Trauma Center, All India Institute of Medical Science, New Delhi, India
Both Australia and India face the challenge of ensuring severely injured people obtain lifesaving interventions, timely treatment of injuries and a restoration of function and independence thereafter. 'Trauma', in this context, refers to physical injury.

The Australia India Trauma Systems Collaboration (AITSC) - funded through the Australia-India Strategic Research Fund (AISRF) Grand Challenge Scheme - seeks to determine better ways of delivering needed care to injured people. The AISRF is a joint initiative between the Indian Government (through the Department of Science and Technology) and the Australian Government (through the Department of Industry, Innovation, and Science) to support the development of strategic alliances between Australian and Indian researchers and fund leading-edge scientific projects between the two countries. Supported projects are designed to lay the foundations for trauma systems development in 
India and improved trauma care in Australia. They will also provide needed evidence about low-cost, trauma system interventions that could be implemented in most countries without the need for major health system redesign. Led by the National Trauma Research Institute (Melbourne) and the JPN Apex Trauma Center at the All India Institute of Medical Sciences (New Delhi), the program brings together some of the world's leaders in trauma care. The AITSC projects include prehospital care, Emergency Department reception and resuscitation, inpatient management and rehabilitation.

This project examined whether computerized decision support would affect resuscitation decisions in a large Indian Trauma Centre.

Timely completion of multiple, rather than single, lifesaving interventions (LSIs) has been demonstrated to be associated with improved outcomes of the severely injured [2]. During the first half-hour of a severely injured patient's arrival at hospital, a lifesaving decision is made every $72 \mathrm{~s}$ [3]. The cognitive and procedural load required of the receiving hospital team, coupled with the condition of the patients and the emotional overlay of bystanders and relatives, can make resuscitation difficult. Errors of omission contributing to patient mortality and morbidity are common. Patient outcomes are variable - and the major variable in resuscitation relates to human factors $[4,5]$.

Up to $58 \%$ of all in-hospital deaths in India have been classified as preventable [6]. Two-thirds of the deaths with injury severity scores of less than 16 have been demonstrated to be preventable and possibly prevented by urgent attention and corrective action. Low-cost interventions such as airway management, fluid resuscitation, haemorrhage control and surgical decision-making protocols have been identified as opportunities for improvement, along with clinical protocols and timely trauma care delivery [6].

The Alfred Hospital in Melbourne, Australia, is a large, Level 1 Adult Trauma Centre. The TRR@ system is a computer-aided, real-time decision support system developed at The Alfred and operational since 2006. The system provides the hospital Trauma Team with computerized clinical decision support for the management of major trauma patients. The TRR (C) system documents real-time interventions and diagnoses and visually prompts the Trauma Team to undertake lifesaving interventions (LSIs) using a large, liquid crystal display. In a randomized, prospective study, TRR@ system usage improved protocol compliance and reduced errors of omission and morbidity. Traumatic shock management errors were reduced by $26 \%$ with an associated one third reduction in the use of blood products [2, 3].

\section{Study Hypothesis}

The purpose of this study was to trial of the introduction of the TRR (C-computerized decision support system to assist resuscitation of the severely injured in a Level 1 Trauma Centre in India. The primary alternative hypotheses for this study were that real-time, computer-assisted, trauma resuscitation decision support would reduce the time taken to initiate LSIs in the first $30 \mathrm{~min}$ of hospital reception and resuscitation.

LSIs were predefined as follows: oxygen administered by mask; respiration assisted with bag-mask ventilation; endotracheal intubation (ETT); recognition of fixed, dilated pupil(s); intercostal catheter insertion (ICC); application of a pressure dressing for uncontrolled haemorrhage; intravenous access (IV); and emergency chest x-ray. Whilst chest x-ray is recommended in ATLS, the timing and the related potential delay to chest decompression are not discussed.

The secondary hypothesis was that real-time vital sign data capture and documentation would be improved using the TRR(C) decision support system [7, 8].

\section{Patients and Methods}

\section{Study Population and Setting}

This was a prospective cohort study with a matched concurrent control group. The study was undertaken at the Jai Prakash Narayan (JPN) Apex Trauma Center, All India Institute of Medical Science (AIIMS), New Delhi, with data collected from 4 April 2017 until 7 July 2017. For study inclusion, patients had to be $\geq 18$ years of age and admitted directly from accident scene into one of two 'Red Triage' resuscitation bays (bay 1 and bay 2 ). Patients transferred from another hospital and patients who were declared deceased on arrival were excluded. There was no inclusion bias regarding gender.

The TRR@ system was installed into bay 2 and was operated by trained staff during daytime hours Monday to Friday 8:00 to 17:00. The control group (without TRRC intervention) consisted of patients allocated into bay 1 at any time. The data extracted from both bays 1 and 2 included date and time, anonymized patient details, vital signs, diagnoses and treatments administered. A project supervisor was at the site to monitor and promote adherence of the intervention during staff meetings.

There was no capacity for randomization and patients were selected based on the availability of the trained staff (who were selected from fulltime clinical and research staff). The study was funded for 3 months of data capture.

\section{Control Group}

The control group were patients treated in bay 1 without the computer-assisted, trauma resuscitation decision support program. The control group represented the 'standard of care', with medical and nursing staff practising according to 
guidelines outlined in the American College of Surgeons Advanced Trauma Life Support program [9]. Documentation was hand-written into the paper-based medical and nursing record which formed the hospital record. Vital signs were also hand-written after looking at the L\&T Star 55 monitor during resuscitation.

The control group data was entered on-line via a purposebuilt REDCap ${ }^{\mathrm{TM}}$ secure web application at the time of the event or retrospectively. Control group notes were often written retrospectively, were not time-logged - and the time of LSIs was often retrospectively estimated. This process was time consuming.

\section{Study Group}

The study group consisted of patients treated in bay 2 where the decision support system had been installed (TRR@ group). Vital sign data, diagnoses and treatment administered during the first 30 min after Trauma Centre arrival were time-logged into the TRR $\odot$ system and subsequently extracted for analyses. Unlike the control group, observations were standardized. Also, the research data collection was automated and required no time from the researchers after the initial $30 \mathrm{~min}$.

For this installation, vital signs were transferred wirelessly from a L\&T Star 55 monitor (Mumbai, India) physiologic monitor system, as well as treatment and diagnostic data via a trained scribe (usually a nurse) using a touch-screen interface. A large LCD display screen, installed above the head of the patient bed, included patient details (age, sex, allergies, weight, mechanism of injury), vital signs, confirmed and unconfirmed diagnoses and treatments administered to the attending Trauma Team. The LCD display screen also displayed 'action prompts' (alerts) to the Trauma Team to assist with decision-making processes and-in particularreduce errors of omission. These advisory prompts could be accepted or declined by Trauma Team Leader, ensuring the final decisions to rest with the treating medical staff. A dedicated scribe was required to interact with the system during resuscitation procedures [2].

The first $60 \mathrm{~min}$ of their resuscitation records were reviewed and the hand-written documentation of resuscitation of the control group was compared to the time-logged, computer-generated records of the study group.

\section{Ethics}

The study was approved as part of the AITSC program by the Institute Ethics Committee All India Institute of Medical Sciences IEC/NP-327/2013 RP-24/2013 OP-3/01.07.2016; the Monash University Humans Research Ethics Committee CF16/18142016000929 and was registered in the Australia New Zealand Clinical Trials Registry ACTRN12618001053213.

\section{Statistical Analyses}

Normally distributed continuous variables are presented using the mean (standard deviation), whilst ordinal or skewed data is presented using the median (inter-quartile range). Error bars for graphical data represent $95 \%$ confidence intervals. Student's $t$ test was used to calculate statistical significance between two means, Wilcoxon Rank Sum test for two medians, the chi-squared test for two proportions, and Fisher's exact test when the count in a two-by-two table cell was less than 5. A $p$ value of $<0.05$ was considered statistically significant. All analyses were performed using Stata version 15.1 (Statacorp, College Station, TX, USA).

\section{Results}

\section{Participants}

For each treatment group, the number of participants enrolled and the subsets of those analyzed for the primary outcome are illustrated in Fig. 1.

Final allocation included 30 patients in the control group (non-TRR@) with a mean age of $31.1(\mathrm{SD}=12.48)$. The study group (TRR@) of 76 patients had a mean age of 38.6 (SD = 13.39). The non-TRR group was $93 \%$ (28/30) male. The TRR group was $86 \%(65 / 76)$ male.

\section{Key Findings}

\section{Vital Sign Observations}

During the first $30 \mathrm{~min}$ of resuscitation, the TRR@ group recorded greater sets of vital signs $(M=5.39$, Median $=5.5$, $\mathrm{SD}=1.77)$ than the control group $(M=1.2$, Median $=1, \mathrm{SD}=$

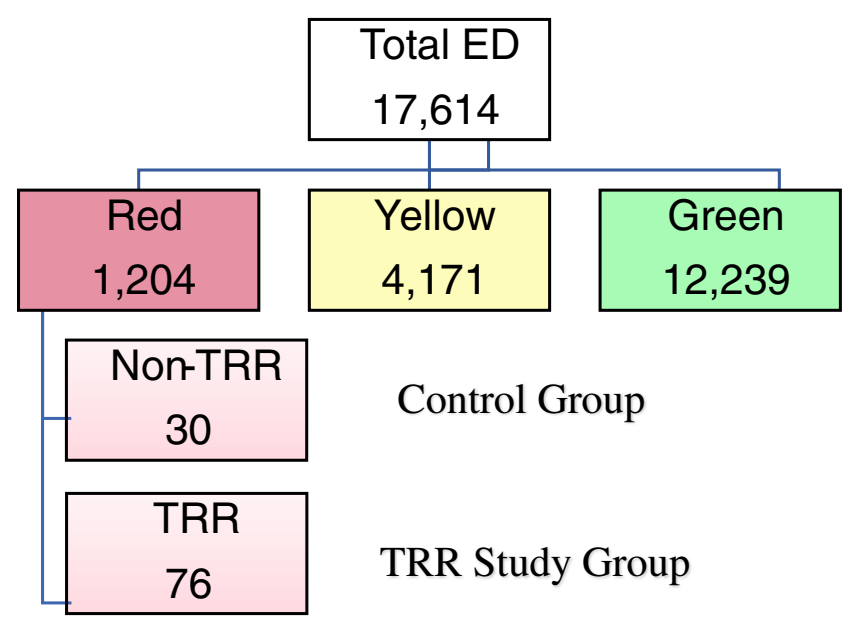

Fig. 1 Total attendances of patients in the emergency department with triage categories and the final sample sizes for analyses 


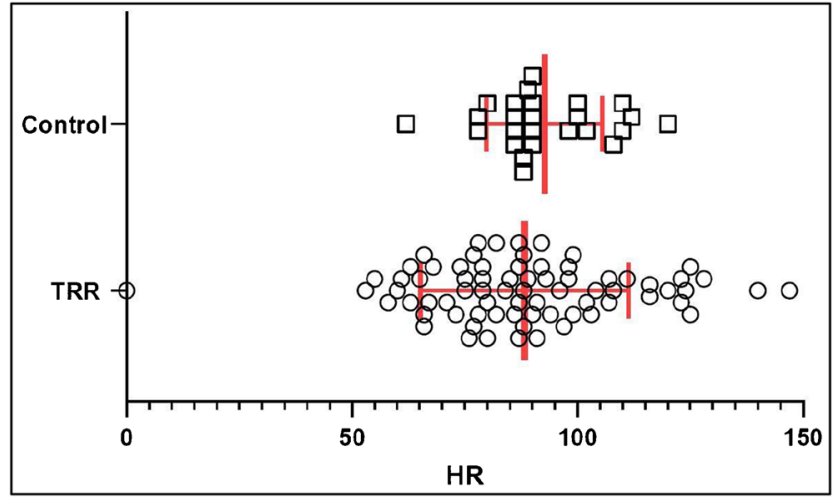

Fig. 2 HR observation time 1

0.71) for HR, BP, RR, GCS and $\mathrm{SpO}_{2}, t(104)=12.54, p<$ 0.0001 .

There was no statistically significant difference between the mean HR for the TRR@ group $(88.23, \mathrm{SD}=23.14, n=$ 73 ) and the control $(M=92.68, \mathrm{SD}=12.88, n=25)$ (Fig. 2). Similarly, no significant difference was observed for SBP (TRR $M=128.2, \mathrm{SD}=28.04, n=70$; controls $M=120.1$, $\mathrm{SD}=17.76, n=25$ ) (Fig. 3).

Despite the sample size difference, observed HR and SBP were distributed proportionally across the two groups (Figs. 3 and 4) indirectly indicating physiologic similarity.

In comparison to one or two sets of vital sign observations for the controls, the TRR $\odot$ system facilitated incremental vital signs capture over the $30 \mathrm{~min}$ (Fig. 4). Consequently, these observations were recorded in the final TRR $\odot$ summary document and a part of medical records.

\section{Lifesaving Interventions}

There were no episodes of CPR, Laryngeal Mask Insertion or Cricothyroidotomy. There were no episodes of intraosseous, venous cutdown or central venous access.

Oxygen was administered in most of the control group as the institutional standard of care. However, $\mathrm{O}_{2}$ administration

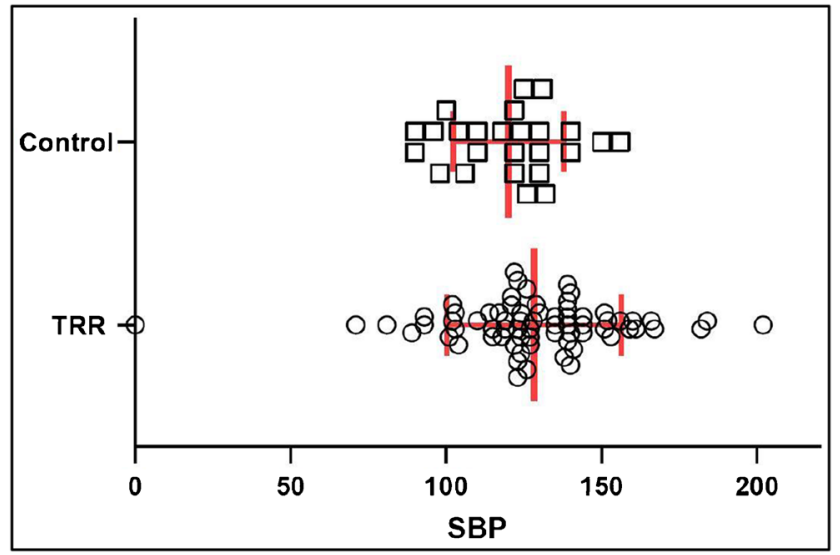

Fig. 3 SBP observation time 1

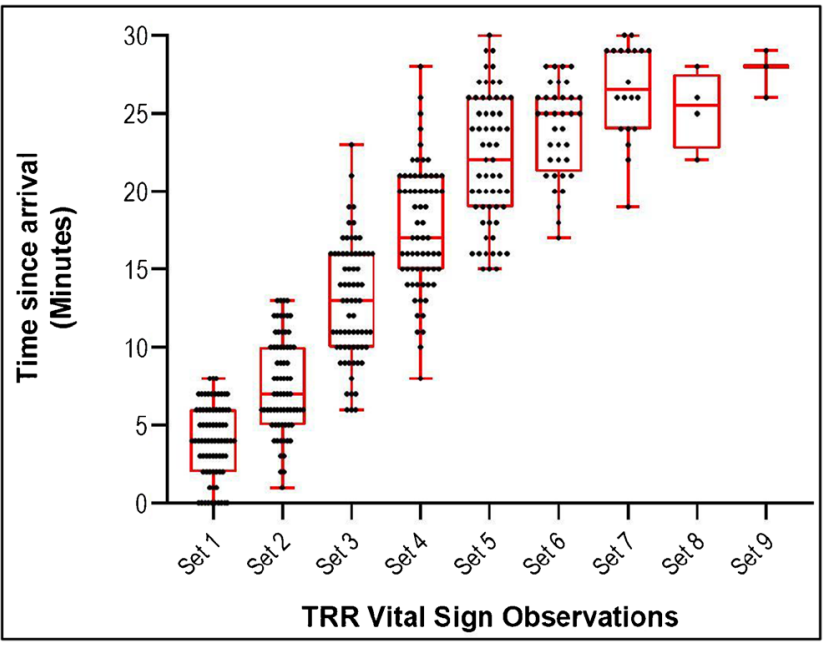

Fig. 4 Frequency of vital signs recorded, represented in medians with $\min / \max$

was prompted only if $\mathrm{SpO}_{2}<93 \%$ in the TRR@ group (Table 1).

There was no difference in the frequency of bag-mask assisted ventilation between the control and TRR@ groups.

Pressure dressings were applied more frequently in the control group. Any wound dressing was termed a 'pressure dressings' in the control group, whilst pressure dressings were prompted only if there was active haemorrhage in the TRR@ group, which made comparison invalid.

Intravenous access was routinely and retrospectively documented at 1 or $2 \mathrm{~min}$ in the control group. However, clinical staff advised that time to IV access as not routinely collected and that the times listed were unreliable for IV access. Time to

Table 1 Lifesaving interventions

\begin{tabular}{|c|c|c|c|}
\hline Lifesaving intervention & Control & TRR & $\begin{array}{l}p \\
\text { value }\end{array}$ \\
\hline $\mathrm{O}_{2}$ administered via mask & $26 / 30$ & $44 / 75$ & 0.007 \\
\hline $\begin{array}{l}\text { Respiration assisted with bag-mask } \\
\text { ventilation }\end{array}$ & $3 / 30$ & $4 / 76$ & 0.38 \\
\hline Endotracheal intubation & $3 / 30$ & $10 / 76$ & 0.76 \\
\hline Mean time (SD) to intubation (min) & $10.7(11.0)$ & $\begin{array}{l}12.0 \\
(4.6)\end{array}$ & 0.089 \\
\hline Pupils noted to be fixed and dilated & $1 / 30$ & $7 / 76$ & 0.44 \\
\hline Intercostal catheter (ICC) & $2 / 30$ & $5 / 76$ & 0.69 \\
\hline Mean time (SD) to ICC (min) & $18.5(9.2)$ & $\begin{array}{l}14.0 \\
(3.6)\end{array}$ & 0.275 \\
\hline Pressure dressing applied & $8 / 30$ & $2 / 76$ & 0.001 \\
\hline Time to IV access (min) & $\begin{array}{l}\text { Not } \\
\text { measured }\end{array}$ & $\begin{array}{l}13.5 \\
(2.4)\end{array}$ & NA \\
\hline Chest x-ray & $9 / 30$ & $22 / 76$ & 0.92 \\
\hline Mean time (SD) to chest $x$-ray (min) & $23.4(6.3)$ & $\begin{array}{l}13.6 \\
(2.4)\end{array}$ & 0.001 \\
\hline
\end{tabular}




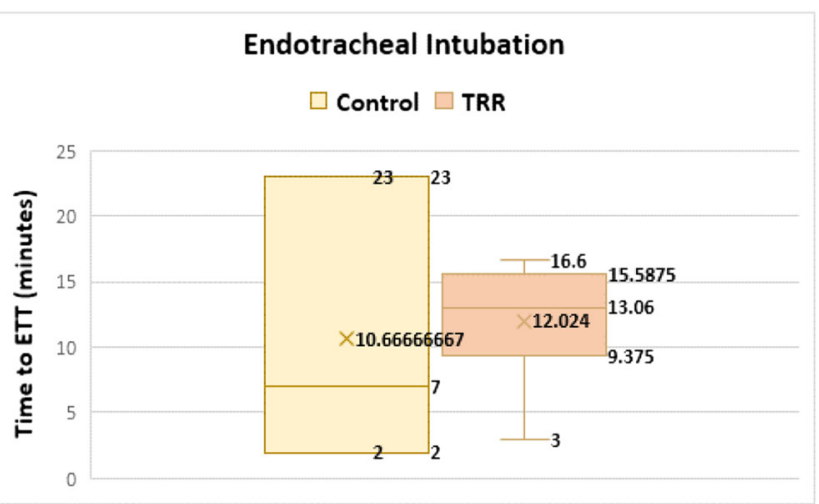

Fig. 5 Time to endotracheal intubation

IV access in the control group was therefore excluded from analyses.

There was no difference in the incidence of patients with fixed, dilated pupils between the groups.

There was no difference in the incidence of endotracheal intubation, intercostal catheter insertion or performance of emergency chest $\mathrm{X}$-ray. These data as well as the vital sign data indicated that the two patient groups were comparable.

There was no difference in the mean times to endotracheal intubation, intercostal catheter insertion or performance of emergency chest x-ray between the control and TRR $($ ) groups. However, with TRR@-computerized decision support, these 3 comparable interventions were performed in a more consistent manner with narrower time distributions (Figs. 5, 6 and 7).

\section{Discussion}

The primary outcome of this study was to determine whether the introduction of the TRRC decision support system into an Indian Trauma Centre would reduce the time to perform previously identified lifesaving interventions. The secondary outcome was to determine if there was improvement in real-time vital sign data capture and documentation.

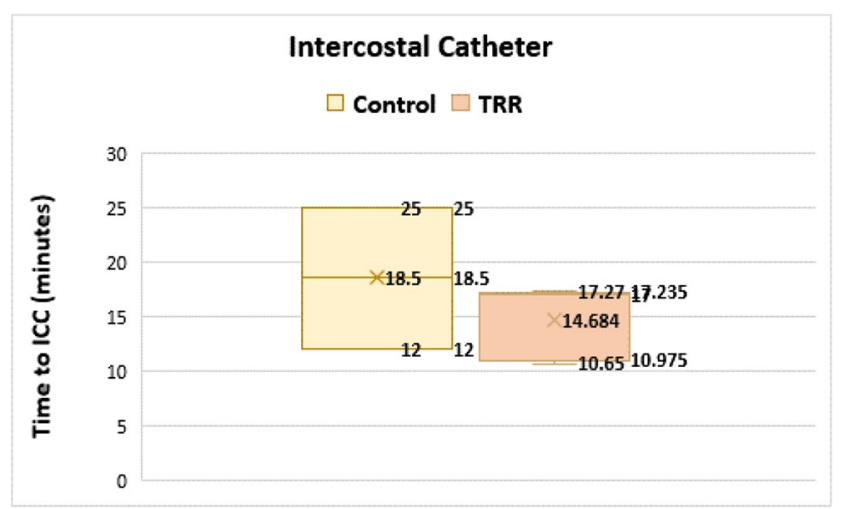

Fig. 6 Time to inter-costal catheter insertion

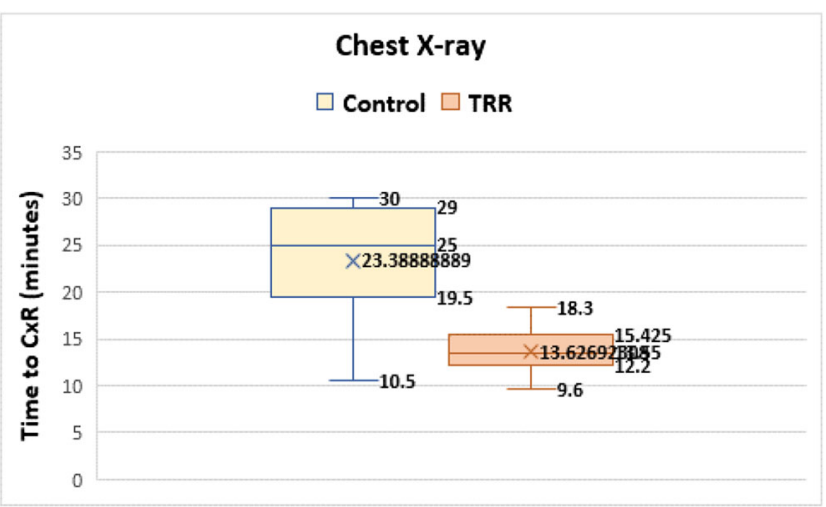

Fig. 7 Time to chest $x$-ray

The key finding was that there was a greater variability in the time taken to perform LSIs in the control group in comparison to the clinicians assisted with the TRR@-computerized decision prompts.

A reduction in time to intervention has been identified as an essential goal in emergency care. The function and fundamental role of any decision support system are to present relevant information and specific recommendations for rapid decisions to be made [10]. The TRR@ group had accurate and realistic documentation of data capture compared to retrospective unreliable manual control data entry. Resuscitation procedures were recorded on reflection of the actual event for the control group and in real time when TRR@ system was in use.

Also, the implementation of the TRR@ system resulted in more frequent vital sign data capture and documentation. Vital sign data are shown to be critical for prioritizing interventions. Furthermore, frequent capture of vital sign datasets provides more information for accurate clinical interpretations, enabled by tools such as clinical decision support systems (CDSS). Previously, CDSS have been integrated with data from electronic health records in an effort to improve vital sign data quality in emergency care [11]. To encourage complete and timely vital sign capture, CDSS should support workflow seamlessly with a direct sense of usefulness. In the case with the TRR@ system, the vital sign data is captured and documented in real time. The vital sign dataset, together with nurses' input of diagnoses and treatment, forms the basis for medical algorithms to be activated for decision-making prompts.

The TRR(C) algorithms and the interface are flexible and were modified for AIIMS's characteristics of injury, trauma care and resuscitation procedure. The system was able to be successfully installed and the staff showed a quick uptake of the technology. It allowed for a detailed data collection and resuscitation analyses which may be used for future training, quality and improvement. The deployment of a computerized decision support system for resuscitation in a Level 1 Trauma Centre in India and its use can be viewed as a demonstration of progress in the field. 
This preliminary study has limitations. This was a small sample size, as the hours of patient recruitment limited patient enrollment (most red area trauma cases arrive after sun down during the warmer months). Furthermore, due to small sample size and a lack of formal trauma registry, the study was not powered to measure mortality. The study was not possible to blind. Randomization was also not possible due to staffing restrictions. However, the patient groups seemed comparable.

Greater than one million people die yearly in India due to trauma. Preventable, early in-hospital deaths require identification of patient subgroups at risk. Prediction models can support clinical judgement, but existing models have methodological limitations [12]. It has been suggested that no single tool for decision-making can adequately target resuscitation and rather, a combination of physiological parameters, communication, cognitive aids and documentation is important for quality care [5, 13]. Human factors such as the time-pressured emergency settings, fatigue and stress can affect decisionmaking capabilities when there is also limited information available. Although the research is limited, cognitive aids have been shown to mediate the deleterious effects of human factors. The TRR@ system addresses such needs by providing the trauma team with vital signs, medically derived 'action prompts' and a visual reference point for team coordination and debriefing.

Author Contributions All authors contributed to the design, implementation and conduct of the trial. Dr Sharma and Mr Jhakal were critically to the training and collection of data at AIIMS. Professor Mark Fitzgerald and Dr Yesul Kim contributed to the majority of manuscript preparation.

Funding This project is part of the Australia-India Trauma Systems Collaboration (www.aitsc.org) funded by the Australian Department of Industry, Innovation, and Science (DIIS) and the Government of India Department of Science and Technology (DST). We would also like to thank Alfred Health for their support of implementing the TRR@ system in India.

Data Availability The datasets generated during the study are available from the corresponding author on reasonable request.

\section{Compliance with Ethical Standards}

Conflict of Interest Mark Fitzgerald has directed the development of the TRR@ system. Yesul Kim is the TRR@ Project Manager. There are no other known conflicts of interest.

Open Access This article is licensed under a Creative Commons Attribution 4.0 International License, which permits use, sharing, adaptation, distribution and reproduction in any medium or format, as long as you give appropriate credit to the original author(s) and the source, provide a link to the Creative Commons licence, and indicate if changes were made. The images or other third party material in this article are included in the article's Creative Commons licence, unless indicated otherwise in a credit line to the material. If material is not included in the article's Creative Commons licence and your intended use is not permitted by statutory regulation or exceeds the permitted use, you will need to obtain permission directly from the copyright holder. To view a copy of this licence, visit http://creativecommons.org/licenses/by/4.0/.

\section{References}

1. World Health Organization. 'Injuries'. http://www.who.int/topics/ injuries/en/ accessed 15 July 2018

2. Mitra B, Bade-Boon J, Fitzgerald MC, Beck B, Cameron PA (2019) Timely completion of multiple life-saving interventions for traumatic haemorrhagic shock: a retrospective cohort study. Burns Trauma 7:22

3. Fitzgerald M, Cameron P, Mackenzie C, Farrow N, Scicluna P, Gocentas R, Bystrzycki A, Lee G, O'Reilly G, Andrianopoulos N, Dziukas L, Cooper DJ, Silvers A, Mori A, Murray A, Smith S, Xiao Y, Stub D, McDermott F, Rosenfeld JV (2011) Trauma resuscitation errors and computer-assisted decision support. Arch Surg 146: $218-225$

4. Fitzgerald MC, Bystrzycki AB, Farrow NC, Cameron PA, Kossmann T, Sugrue ME, Mackenzie CF (2006) Trauma reception and resuscitation. ANZ J Surg 76:725-728

5. Groombridge CJ, Kim Y, Maini A, Smit V, Fitzgerald MC (2019) Stress and decision-making in resuscitation: a systematic review. Resuscitation. 144:115-122

6. Roy N, Kizhakke Veetil D, Khajanchi MU, Kumar V, Solomon H, Kamble J et al (2017) Learning from 2523 trauma deaths in Indiaopportunities to prevent in-hospital deaths. BMC Health Serv Res $17: 142$

7. Eastman AB (2011) Are computers a new member of the trauma team? Arch Surg 146:225

8. Anazodo AN, Murthi SB, Frank MK, Hu PF, Hartsky L, Imle PC, Stephens CT, Menaker J, Miller C, Dinardo T, Pasley J, Mackenzie CF (2015) Assessing trauma care provider judgement in the prediction of need for life-saving interventions. Injury. 46:791-797

9. American College of Surgeons Advanced Trauma Life Support Program. https://www.facs.org/quality-programs/trauma/atls accessed 10 July 2019

10. Bashiri A, Savareh BA, Ghazisaeedi M (2019) Promotion of prehospital emergency care through clinical decision support systems: opportunities and challenges. Clin Exp Emerg Med 6:288296

11. Skyttberg N, Vicente J, Chen R, Blomqvist H, Koch S (2016) How to improve vital sign data quality for use in clinical decision support systems? A qualitative study in nice Swedish emergency departments. BMC Med Inform Decis Mak 16:61

12. Liu NT, Holcomb JB, Wade CE, Salinas J (2015) Improving the prediction of mortality and the need for life-saving interventions in trauma patients using standard vital signs with heart-rate variability and complexity. Shock. 43:549-555

13. Gerdin M, Roy N, Khajanchi M, Kumar V, Dharap S, FellanderTsai L et al (2014) Predicting early mortality in adult trauma patients admitted to three public university hospitals in urban India: a prospective multicentre cohort study. PLoS One 9:e105606

Publisher's Note Springer Nature remains neutral with regard to jurisdictional claims in published maps and institutional affiliations. 